Preprint typeset using $\mathrm{LAT}_{\mathrm{E}} \mathrm{X}$ style emulateapj v. 2/16/10

\title{
ORBITAL SUPPORT OF FAST AND SLOW INNER BARS IN DOUBLE BARRED GALAXIES
}

\author{
Witold Maciejewski and Emma E. Small \\ Astrophysics Research Institute, Liverpool John Moores University, Twelve Quays House, Egerton Wharf, Birkenhead, CH41 1LD
}

\begin{abstract}
We analyze how the orbital support of the inner bar in a double-barred galaxy (nested bars) depends on the angular velocity (i.e. pattern speed) of this bar. We study orbits in seven models of double bars using the method of invariant loops. The range of pattern speed is covered exhaustively. We find that not all pattern speeds are allowed when the inner bar rotates in the same direction as the outer bar. Below a certain minimum pattern speed orbital support for the inner bar abruptly disappears, while at high values of this speed the orbits indicate an increasingly round bar that looks more like a twist in the nuclear isophotes than a dynamically independent component. For values between these two extremes, orbits supporting the inner bar extend further out as the bar's pattern speed decreases, their corresponding loops become more eccentric, pulsate more, and their rotation becomes increasingly non-uniform, as they speed up and slow down in their motion. Lower pattern speeds also lead to a less coherent bar, as the pulsation and acceleration increasingly varies among the loops supporting the inner bar. The morphologies of fast and slow inner bars expected from the orbital structure studied here are recently recovered observationally by decomposition of double barred galaxies. Our findings allow us to link the observed morphology to the dynamics of the inner bar.
\end{abstract}

Subject headings: methods: analytical — stellar dynamics — galaxies: kinematics and dynamics galaxies: nuclei - galaxies: spiral — galaxies: structure

\section{INTRODUCTION}

Double barred galaxies are barred spiral galaxies that contain an additional small bar nested within the main bar. First observed by de Vaucouleurs (1975), they may constitute $\sim 30 \%$ of barred galaxies, as implied by photometric surveys (Erwin \& Sparke 2002, Laine et al. 2002). However, cross-correlation of these surveys suggests a lower percentage (Moiseev 2010), which is consistent with a kinematic survey of double barred candidates using integral field spectroscopy (Moiseev et al. 2004). The frequency at which double bars are observed indicates that they are either recurrent or long-lived phenomena. Since inner bars occur often in early-type galaxies with little or no gas to drive evolution, they are most likely long-lived. This is consistent with the fact that inner bars are detected in both optical and IR observations, indicating that they are stellar structures. Observations of the apparent random orientations of the two bars suggest that the bars are rotating independently (Buta \& Crocker 1993; Friedli \& Martinet 1993). This was later confirmed for the galaxy NGC 2950, whose inner and outer bar do not rotate at the same pattern speed (Corsini et al. 2003).

It is difficult to understand how two independently rotating nested bars can be maintained by regular motions of stars to create a long-lived stable system. In particular, the resonances of one bar will interfere with the orbital support of the other, which is likely to produce chaotic motions and thus constrain possible parameters of stable self-consistent double bars. However, when studying the orbital response to an assumed potential of two independently rotating bars Maciejewski \& Sparke (2000, hereafter MS00) found stable orbits that can support each bar in its rotation. Maciejewski \& Athanas-

wxm@astro.livjm.ac.uk soula (2007, hereafter MA07) showed that double bars are sustained by families of stable double frequency orbits, i.e. orbits that oscillate only with the driving frequencies of the two bars. Support for the two bars is provided by trajectories trapped around these double frequency orbits.

Maciejewski \& Athanassoula (2008, hereafter MA08) studied the trapping of trajectories around regular orbits in 23 models of double bars, by varying the parameters characterising both bars. They varied the lengths, masses and eccentricities of the bars, but they found that out of the parameters of the inner bar, its pattern speed affected the trapping most. In this paper we investigate how the appearance of double frequency orbits supporting the inner bar changes with its pattern speed. Thus we perform the orbital structure studies, following the method developed by Contopoulos \& Papayannopoulos (1980), Athanassoula et al. (1983), Teuben \& Sanders (1985) and others. These studies do not aim to construct self-consistent models, but rather explore changes in the system that occur when its main parameters vary.

We study orbits in Models $01-05$ from MA08, where the pattern speed of the inner bar was varied between 80 $\mathrm{km} \mathrm{s}^{-1} \mathrm{kpc}^{-1}$ and $120 \mathrm{~km} \mathrm{~s}^{-1} \mathrm{kpc}^{-1}$. We also construct new models $02 \mathrm{E}$ and $05 \mathrm{E}$ here, which extend this range down to $70 \mathrm{~km} \mathrm{~s}^{-1} \mathrm{kpc}^{-1}$ and up to $140 \mathrm{~km} \mathrm{~s}^{-1} \mathrm{kpc}^{-1}$. Thus all models considered in this paper are identical except for the pattern speed of the inner bar. They are the same as Model 2 from MS00, where the semi-major axis of the outer bar is $6 \mathrm{kpc}$ and the semi-major axis of the inner bar is $1.2 \mathrm{kpc}$. The axial ratios of the outer and inner bar are 2.5 and 2.0, respectively. The mass of the inner bar is $15 \%$ of the mass of the outer bar. The pattern speed of the outer bar is $36 \mathrm{~km} \mathrm{~s}^{-1} \mathrm{kpc}^{-1}$ and both bars rotate in the direction of the stellar rotation in the disk. 
In Section 2, we briefly review the method that uses invariant loops in the search for regular orbits in double bars, and we describe how we derive the position angle and eccentricity of the loops as well as the extent to which they support the inner bar. In Section 3, we analyze the orbital structure in each of the seven models, and we find what trends in the parameters and behaviour of the inner bar, as a function of its pattern speed, its orbital structure indicates. We discuss the validity of orbital structure studies in Section 4, where we also attempt to obtain information on the dynamics of the inner bar from its observed characteristics. We summarize our findings in Section 5.

\section{METHOD}

Like stable closed periodic orbits in a single bar, stable double frequency orbits in double bars provide support for both bars by trapping trajectories that oscillate around them. The difficulty in studying double frequency orbits within two independently rotating bars is that such a potential oscillates in time, and these orbits do not close. However, double frequency orbits can be visualised using the concept of a loop, invented by Maciejewski \& Sparke (1997). A coherent tutorial on the method based on invariant loops can be found in MA07 and Maciejewski (2010); here we outline its basic concepts. Any orbit (which consists of particle positions over a continuum of moments in time) can be represented for any instantaneous shape of the oscillating potential by points on this orbit plotted at the moments when the potential repetitively takes that given shape (a discreet set of moments in time). In particular, double frequency orbits are represented by closed curves, called loops (MA07). The loops change shape as the bars rotate through each other, responding to the oscillating potential, but they regain their former shape when the bars return to their former relative orientation. The shape of the loop does not depend of the reference frame in which the loop is drawn, hence the loops are sometimes called the invariant loops.

Trajectories trapped around a double frequency orbit are represented by rings that enclose the loop which represents that orbit. Thinner rings indicate smaller amplitudes of oscillations and a more tightly bound trajectory (MA07). MA08 constructed ring-width diagrams, which display the thickness of the rings as a function of the trajectory's initial position along the minor axis of the aligned bars and initial velocity along the major axis. Trajectories in the plane of the galactic disk can be determined from these two starting conditions if it is assumed that they begin on the minor axis with no radial velocity component. MA08 showed that this subset of initial conditions reproduces all major families of regular orbits in double bars.

Figure 1 displays the ring-width diagrams for all the models of double barred galaxies analysed in this paper. These are models $01-05$ from MA08, whose ring-width diagrams have been presented there, and new models $02 \mathrm{E}$ and $05 \mathrm{E}$, whose ring-width diagrams are constructed here using the same algorithm. The darker regions represent smaller ring-widths, hence initial conditions from the darkest spine of the two arches generate a close approximation to double frequency orbits. The outer arch corresponds to orbits that support the outer bar and orig- inate from the $x_{1}$ orbits in a single bar. The inner arch corresponds to orbits that originate from the $x_{2}$ orbits of a single (outer) bar. Loops that represent these orbits vary between those which are perpendicular to the outer bar, and those which support the inner bar, with continuous transition between these two extremes. Because of this continuity, these orbits belong to a single family, which we call the $x_{2}$ family. Note that loops which support the inner bar (sometimes vaguely called 'the inner bar's $x_{1}$ orbits') are called in our notation the $x_{2}$ loops, which reflects their origin.

In order to recover the orbits supporting the inner bar, we sample the inner arch uniformly along the horizontal axis of the diagram (which is proportional to the square root of the initial position coordinate), choosing initial conditions that return the smallest ring-widths. These initial conditions, marked in Figure 1 by red dots, only approximate the double frequency orbits, with accuracy proportional to the resolution of the ring-width diagram. This accuracy is sufficient for the purpose of this paper. Loops representing such approximate double frequency orbits are constructed by following a particle on this orbit for 399 successive realignments of the bars. Thus each loop is made up of 400 points. For each of the models, loops whose initial conditions are marked by red dots in Figure 1 are plotted in Figure 2 for three different relative angles between the two bars in the imposed potential, and diagrams characterizing properties of these loops are presented in Figures 3 and 4.

\subsection{Determination of the position angle (PA) of the loops}

We define the position angle (PA) of the semi-major axis of each loop as the PA at the maximal radial coordinate on this loop. This method works well when loops are close to elliptical $(\mathrm{m}=2$ component dominating) but will return incorrect semi-major axes for loops that are more rectangular $(\mathrm{m}=4$ component dominating $)$ or that are not symmetrical at all. However all the loops analysed here appear to be very ellipse-like. To test the validity of this analysis we reflect the points of the inner most and most circular loop of model 01 through its derived semi-major axis. The innermost loop was chosen in order to ensure it was symmetrical since it appears to be affected by both bars and is aligned with neither. For both orbits there was a small deviation in the reflected points compared to the original, but it was of the order of the width of the ring that best approximates its loop. This confirms the validity of the method. The method is subject to error arising from insufficient sampling or scattering of points near the real semi-major axis. We estimate the error as the difference between the two estimates of the PA of the semi-minor axis: one being the $\mathrm{PA}$ at the minimum radial coordinate, and the other the PA of the semi-major axis subtracted by $90^{\circ}$. The error is expected to be greater for more circular loops, where the estimation of the semi-major axis is more affected by scatter. The PAs for all the loops in each model, together with estimated error are plotted in the left panels of Figure 3 for four relative positions of the bars in the imposed potential: $0^{\circ}, 45^{\circ}, 90^{\circ}$ and $135^{\circ}$.

\subsection{Determination of the eccentricity of the loops}



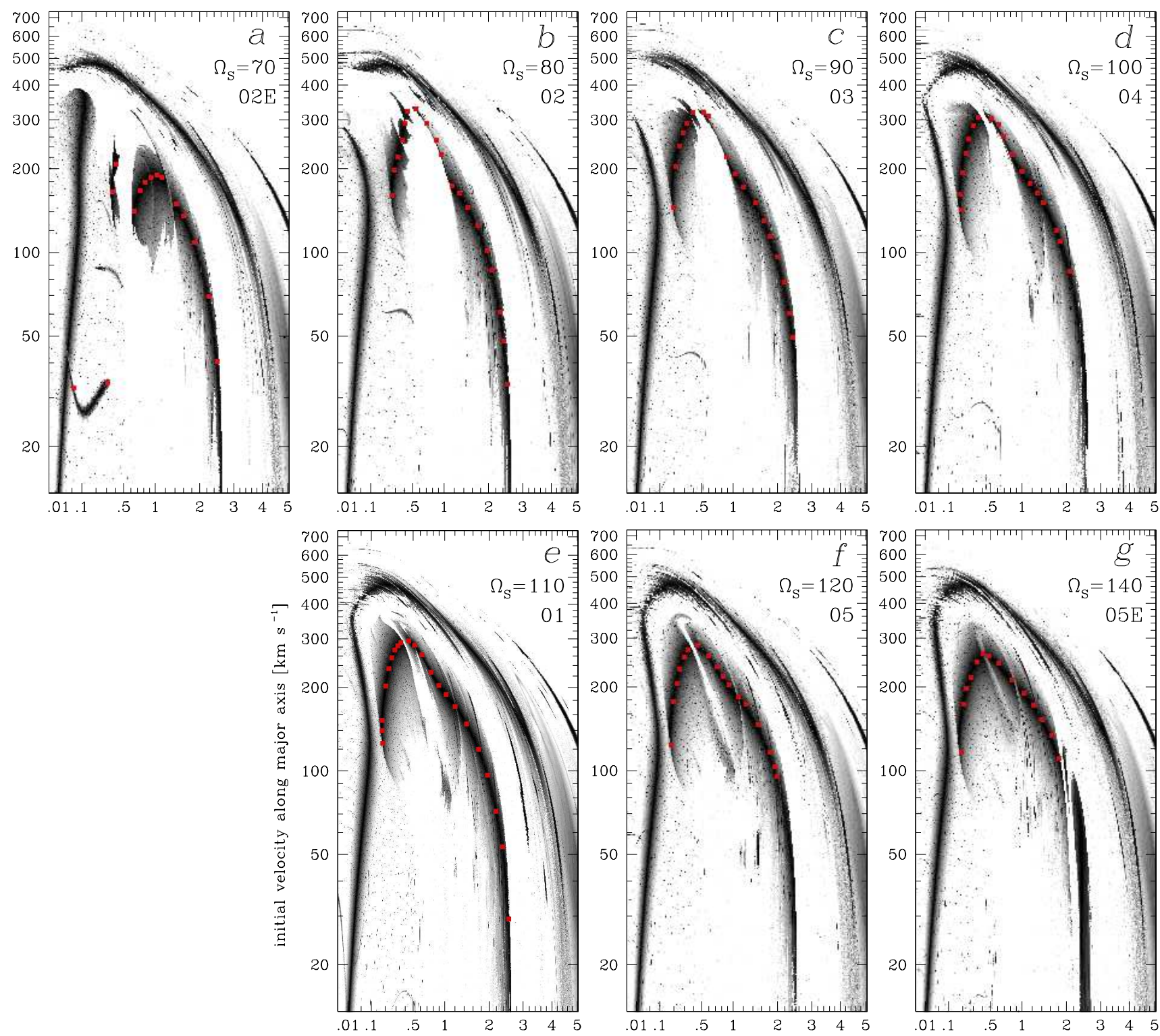

initial position on minor axis $[\mathrm{kpc}$

Figure 1. Diagrams of ring-width for all the models (02E, 02, 03, 04, 01, 05 and 05E - ordered by increasing pattern speed of the inner bar) as a function of initial position along the minor axis of the aligned bars (horizontal axis) and the initial velocity along the major axis (vertical axis). Darker regions represent smaller ring-widths. The red points correspond to the initial conditions of representative $x_{2}$ loops plotted in Figure 2, and analysed in Figures 3 and 4 . In the top-right corner of each frame, we list the pattern speed of the inner bar $\left(\Omega_{S}\right.$, in $\mathrm{km} \mathrm{s}^{-1} \mathrm{kpc}^{-1}$ ), and the model number.

The eccentricity of a loop is quantified by its axial ratio: the ratio between the lengths of its semi-major and semi-minor axes. The semi-minor axis here is taken as the radial coordinate of the point located $90^{\circ}$ away from the previously determined semi-major axis. Unlike the $\mathrm{PA}$, the actual lengths of the semi-major and semi-minor axes are not appreciably affected by the scatter and the error in axial ratio is small. The axial ratios for all loops in each model are plotted in the right panels of Figure 3 for four different relative positions of the bars in the imposed potential: $0^{\circ}, 45^{\circ}, 90^{\circ}$ and $135^{\circ}$. The axial ratios at $45^{\circ}$ and $135^{\circ}$ are the same, which reflects the symmetry of the problem upon time reversal. The amplitude at which the loops pulsate can be estimated from the variation in eccentricity of the loops as the bars rotate. From Figure 2, we see that the eccentricity of the loops is smallest when the bars are aligned and largest when the bars are perpendicular. The ratios of eccentricity of loops, when the bars in the imposed potential are separated by $45^{\circ}$ and $90^{\circ}$, to their eccentricity, when the bars are aligned, are displayed for all models in Figure 4.

\subsection{Determination of the length of the inner bar}

Although by assuming the potential in which we calculate the orbits we thereby set the lengths of the two bars, we have to analyse the orbital response in order to estimate to what extent the orbits support the bars. Loops that represent orbits supporting a given bar should follow 

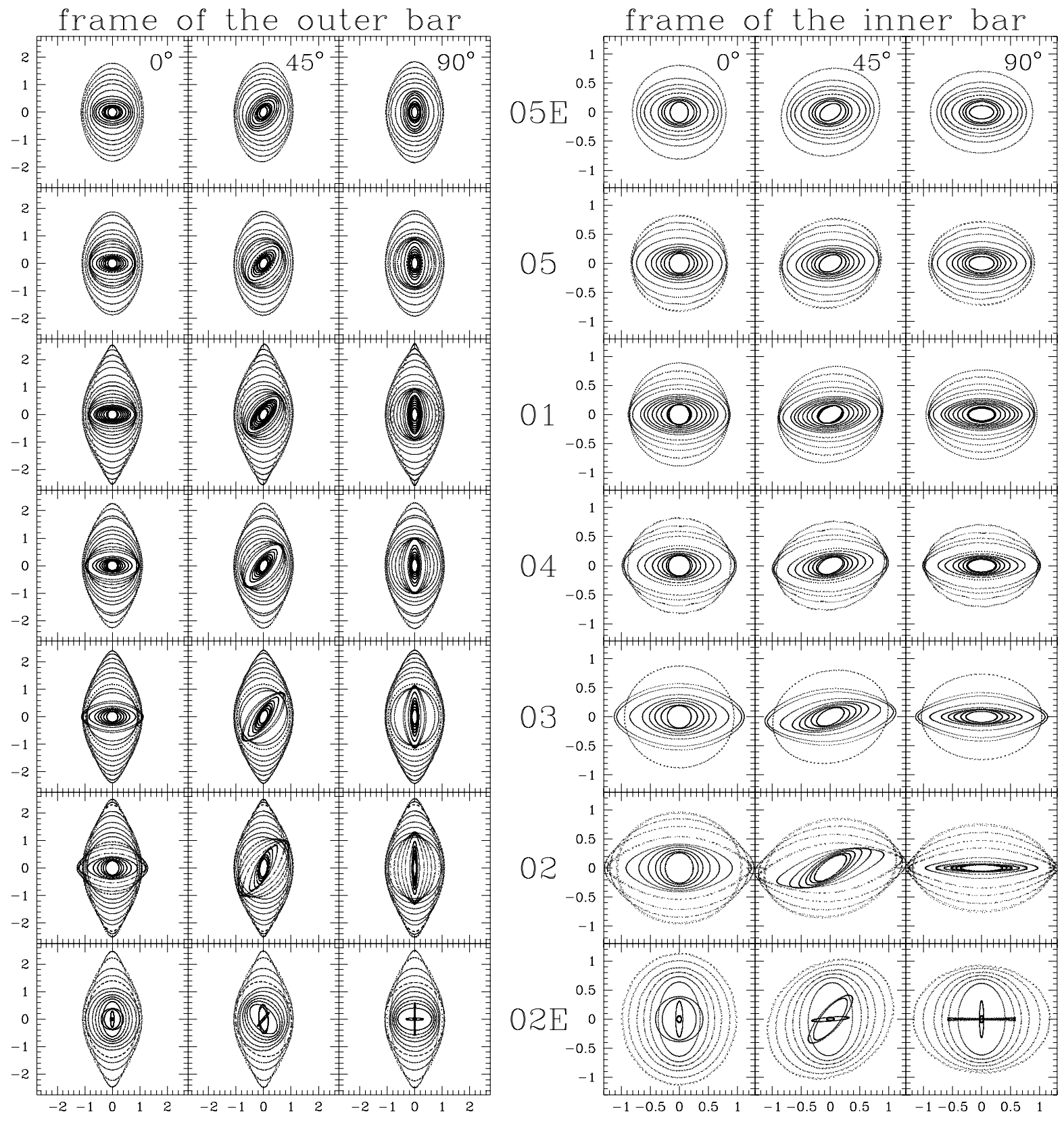

Figure 2. Representative $x_{2}$ loops in all models, whose initial conditions are marked by the red dots in Figure 1 . The loops in the three left-hand columns are plotted in the frame of the outer bar, in which the outer bar remains horizontal and the inner bar rotates anticlockwise. In the three right-hand columns, the inner loops out to the most circular loop are plotted in the frame of the inner bar, where the inner bar remains horizontal. For each reference frame the 1st, 2nd and 3rd panels in each row show loops when the angle between the bars in the imposed potential is $0^{\circ}, 45^{\circ}$ and $90^{\circ}$, respectively. The rows show, from top to bottom, models $05 \mathrm{E}, 05,01,04$, 03, 02 and 02E. The units on the axes are kpc.

that bar as the bars rotate through each other. For most models in Figure 2, the inner $x_{2}$ loops generally do seem to follow the inner bar, while the outer $x_{2}$ loops remain perpendicular to the outer bar. There is a smooth transition between the inner and outer $x_{2}$ loops, where loops are almost circular. This is confirmed by the PA plots in the left-hand panels of Figure 3, where the PAs of the outer loops remain consistently at $90^{\circ}$ regardless of the relative position of the bars, while the PAs of loops with semi-major axes roughly between $0.3 \mathrm{kpc}$ and $0.8 \mathrm{kpc}$ or more (depending on the model) are also consistent with each other, but their value changes in accordance with the rotation of the inner bar in this frame. Departures from this simple picture, caused by different behaviour of up to the three most inner loops in all models and by incoherent PAs of inner loops in models with lower inner bar's pattern speeds, will be analyzed in Section 3.1.

In Figure 1, the initial conditions for the $x_{2}$ loops come from the darkest spine of the inner arch, and therefore the loops can be ordered into a sequence along this arch. This sequence is reflected in Figure 3 by lines which connect points marking individual loops. We determine the 

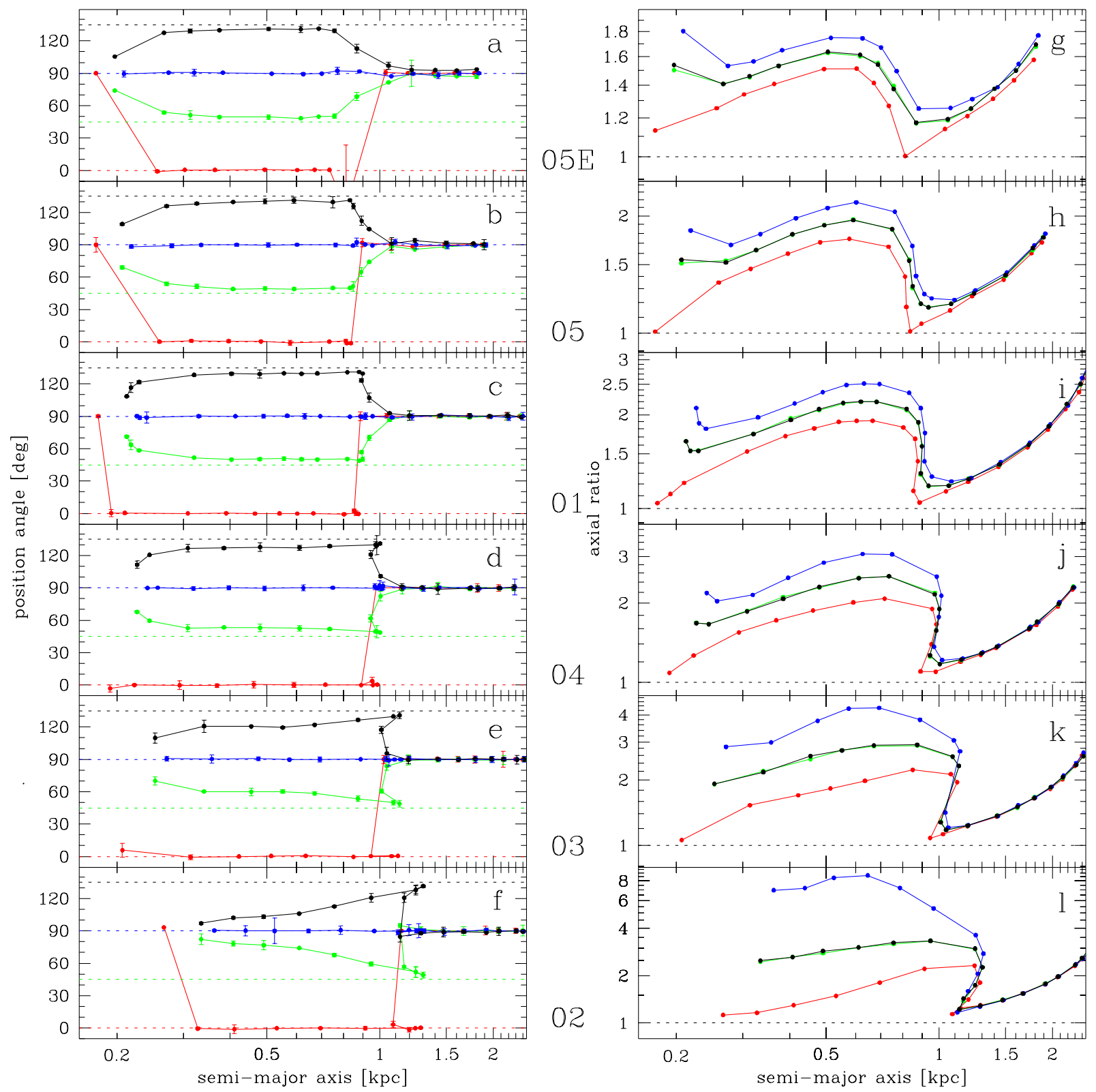

Figure 3. For all representative $x_{2}$ loops in models 05E, 05, 01, 04, 03 and 02 (top to bottom), the position angle (PA, in degrees) in the frame corotating with the outer bar in which that bar remains horizontal (left-hand panels) and the axial ratio (right-hand panels) are plotted against the length of the semi-major axis (in kpc). Plots are for the relative angle between the bars in the imposed potential equal $0^{\circ}$ (red), $45^{\circ}$ (green), $90^{\circ}$ (blue) and $135^{\circ}$ (black). Lines that connect points representing loops reflect the sequence along the arch in Figure 1.

last loop supporting the inner bar as the last of the loops that maintain a consistent PA, which varies in accordance with the PA of the inner bar in the imposed potential. Then among the loops supporting the inner bar we find the one whose major axis is longest, and the length of the bar is defined as the length of this major axis. Note that the loop with the longest major axis does not have to be the last one in the sequence of loops supporting the bar. As can be seen in Figure 3, in models with lower angular velocity of the inner bar (lower panels), the semi-major axis of the loops which support that bar initially increases along the sequence defined by the arch in Figure 1, but then reaches a maximum and de- creases, so that the last of the loops supporting the bar is not the loop of the longest semi-major axis.

Since the loops presented here are only a representative sample of the $x_{2}$ orbital family, the definition formulated above underestimates the length of the inner bar. The upper limit for this length can be estimated in two ways, depending on whether the loop with the longest semimajor axis is the last one in the sequence of loops supporting the bar. If it is, then the bar is shorter than the semi-major axis of the next loop in the sequence past the last one supporting the bar. Otherwise, the upper limit for the bar length is calculated by adding to the longest among the semi-major axes of loops that support the bar 
Table 1

Characteristics of models of double bars

\begin{tabular}{|c|c|c|c|c|c|c|c|}
\hline Model name & $\Omega_{S}\left[\mathrm{~km} \mathrm{~s}^{-1} \mathrm{kpc}^{-1}\right]$ & $r_{C R}[\mathrm{kpc}]$ & $a_{0}[\mathrm{kpc}]$ & $a_{0} / r_{C R}$ & $e_{0}^{\max }$ & $e_{90} / e_{0}$ & $\delta P A$ [degrees] \\
\hline 02 & 80 & 2.92 & 1.284 & 0.440 & 2.32 & $3.68(1.46-6.46)$ & $18.55(3.63-33.12)$ \\
\hline 03 & 90 & 2.63 & 1.117 & 0.425 & 2.23 & $1.88(1.39-2.33)$ & $10.82(3.92-15.42)$ \\
\hline 04 & 100 & 2.39 & 0.984 & 0.412 & 2.08 & $1.40(1.27-1.53)$ & $6.44(3.72-8.34)$ \\
\hline 01 & 110 & 2.19 & 0.877 & 0.401 & 1.91 & $1.29(1.23-1.32)$ & $5.26(3.88-6.70)$ \\
\hline 05 & 120 & 2.01 & 0.812 & 0.403 & 1.75 & $1.23(1.20-1.25)$ & $5.47(3.66-9.09)$ \\
\hline $05 \mathrm{E}$ & 140 & 1.74 & 0.735 & 0.422 & 1.51 & $1.18(1.15-1.22)$ & $5.20(3.21-8.73)$ \\
\hline
\end{tabular}

Note. - Col.2: pattern speed of the inner bar in the assumed potential (i.e. the input parameter of the model). Col.3: corotation radius of the inner bar. Col.4: the length of the semi-major axis of the inner bar as determined from the extent of the loops that support it, measured at the moment when the two bars are parallel. Col.5: the ratio of the length of the inner bar to its corotation radius. Col.6: the largest axial ratio of the loops supporting the inner bar at the moment when the two bars are parallel. Col.7: average ratio of the eccentricity of the loops supporting the inner bar at the moment when the two bars are perpendicular to the eccentricity when the two bars are parallel (in parenthesis the range of this ratio for all the loops that support the inner bar). Col.8: the average offset of the PA of the loops supporting the inner bar from the PA of the inner bar in the assumed potential being $45^{\circ}$ (in parenthesis the range of this offset for all the loops that support the inner bar).

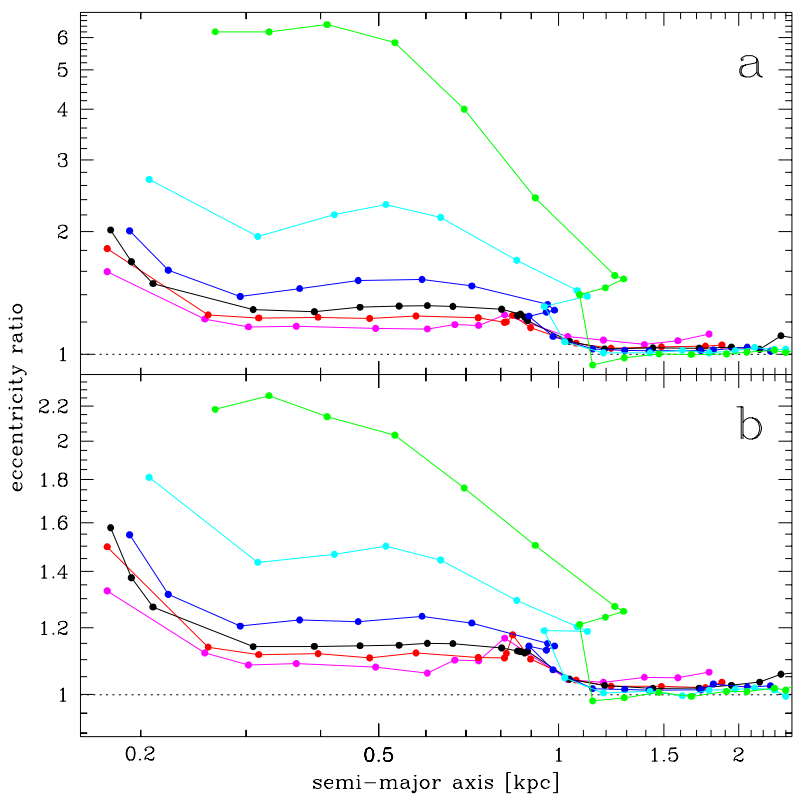

Figure 4. The ratio of eccentricity of the loops, when the angle between the bars in the imposed potential is $90^{\circ}$ (top panel) and $45^{\circ}$ (bottom panel), to their eccentricity when the bars are aligned, plotted against their semi-major axes measured when the bars are aligned. Each representative loop in models 02-05E is marked by a point, with model $05 \mathrm{E}$ being plotted in pink, 05 - in red, $01-$ in black, 04 - in blue, 03 - in cyan and 02 - in green. Lines that connect points representing loops reflect the sequence along the arch in Figure 1.

an average spacing in the semi-major axis between the loops that precede and follow it.

\section{RESULTS}

There is a gradual change in the global orbital structure of the calculated models, as the angular velocity of the inner bar is varied. These global properties are quantified and listed in Table 1 for all models in which the inner bar has orbital support. The pattern speed of the inner bar, listed in the second column, is the input parameter of the model. The corotation radius of the inner bar, listed in the third column, is calculated in the axisymmetric approximation, and therefore depends only on the radial mass distribution in the model. The parameters listed in columns $4-8$ are extracted from the orbital structure calculated for every model. The variation of these parameters with the pattern speed of the inner bar is studied in the second half of this section, while in the first half we remark on the details of finding regular orbits in each model, and on extracting from them the numerical parameters for Table 1.

\subsection{Notes on individual models}

\subsubsection{Model 01}

We start with model 01 because it is the reference model in MA08 and MS00. The two bars in model 01 are in resonant coupling with the corotation of the inner bar overlapping the Inner Lindblad Resonance (ILR) of the outer bar. MA08 calculated the ring-width diagram for this model with the resolution twice higher than that for the other models (i.e. twice denser sampling of the initial conditions on both axes), therefore the parameters of the loops can be determined here more accurately, giving closer approximations to double frequency orbits and hence smoother loops in Figure 2. On the example of model 01 we show here how the parameters of the loops are extracted.

The last loop supporting the inner bar in model 01 is the 12 th loop. According to the definition adopted in Section 2.3, the length of the inner bar is estimated by the longest major axis among the loops that support it. In model 01 it is also the 12 th loop. It is evident from the PA diagram in Figure 3c that almost all $x_{2}$ loops, up to the radial extent determined above, are well aligned with each other for each of the four relative positions of the bars in the imposed potential. This indicates that the bar maintains its shape coherently throughout rotation. However, the three inner-most loops are not aligned with the loops supporting the inner bar, when the angle between the two bars in the imposed potential is $45^{\circ}$ and $135^{\circ}$. The acceleration of these loops is more extreme and, as we see below, the inner-most loop is actually dominated by pulsation. The initial conditions for these loops were taken from the edge of the left leg of the inner arch in the ring-width diagram (Figure 1e). They are close to the inner ILR of the outer bar and are most likely affected by this resonance. Since the inner-most regions of the inner bar can only be supported when the $x_{2}$ orbits extend to the galaxy centre, there may be no inner ILR in self-consistent double barred galaxies, and the behaviour of the innermost loops in model 01 is likely to be 
an artifact caused by the potential assumed in this work. Below we exclude the inner-most loops when estimating the PA and pulsation of the inner bar.

The acceleration and pulsation of the loops supporting the inner bar, previously seen by MS00, is clear in the right-hand panels of the 3rd row in Figure 2. These loops are aligned with the inner bar in the imposed potential when the angles between the bars there are $0^{\circ}$ and $90^{\circ}$ but they lead when this angle is $45^{\circ}$ and trail when it is $135^{\circ}$. This is confirmed by the PA diagram in Figure $3 \mathrm{c}$ : when the angle between the two bars in the imposed potential is $45^{\circ}$, the mean PA of the loops supporting the inner bar in model 01 (loops $4-12$ ) is $50.3^{\circ}$ with average error $\sim 1.1^{\circ}$. The pulsation of the inner bar is evident in Figure 2 from the change in the appearance of the loops between the time when the bars are parallel and perpendicular to each other. The loops are most eccentric when the bars are perpendicular. This is also shown in Figure 3i: the axial ratio of the loops supporting the inner bar increases when the two bars are not aligned. These loops seem to be pulsating consistently since their eccentricities change at the same rate (Figure 4), aside from the three innermost loops, which strongly pulsate.

The transition between loops supporting the inner bar and those perpendicular to the outer bar occurs for initial conditions located in a very smooth part of the inner arch in the ring-width diagram (Figure 1e), between initial positions along the minor axis of 0.65 and $1.2 \mathrm{kpc}$. At smaller initial positions there is a discontinuity in the inner arch. MA08 suggested that this feature was associated with the transition region, but it occurs between the 9 th and 10th loop, which is in the middle of the orbits supporting the inner bar, and its origin is still unclear.

\subsubsection{Model 05}

The pattern speed of the inner bar in models 05 and $05 \mathrm{E}$ is successively higher than in model 01 . In model 05 it is $120 \mathrm{~km} \mathrm{~s}^{-1} \mathrm{kpc}^{-1}$. In this model, the loops supporting the inner bar do not extend as far as they do in model 01. The last loop supporting the inner bar is the 8th loop, and this loop also has the longest semi-major axis out of the loops that support the inner bar. As in model 01, the loops supporting the inner bar behave coherently in this model, which can be seen in Figure $3 \mathrm{~b}$. The inner-most loop is misaligned and dominated by pulsation and is therefore excluded from supporting the inner bar. The mean PA of the loops that support the inner bar (loops 2-8) when the bars in the imposed potential are separated by $45^{\circ}$ is $50.5^{\circ}$ with an average error of $1.6^{\circ}$. This implies the acceleration of the inner bar in model 05, within the errors, the same as in model 01. The axial ratio plot in Figure 3 h shows that the loops are rounder in model 05 than in model 01. Variation in eccentricity of the loops (Figure 4, Table 1) implies that the inner bar in model 05 pulsates less than in model 01.

\subsubsection{Model 05E}

The pattern speed of the inner bar for this model is $140 \mathrm{~km} \mathrm{~s}^{-1} \mathrm{kpc}^{-1}$. This is a new model, not presented in MA08, and its ring-width diagram is shown in Figure $1 \mathrm{~g}$. The discontinuity at the top of the inner arch in this diagram is reduced when compared to the other models. There is also more grey area surrounding the inner arch, which indicates a greater phase-space volume occupied by trapped orbits that can provide support for the inner bar. In this model, the loops supporting the inner bar are completely nested within the loops that are perpendicular to the outer bar. There is no gathering of loops at the end of their semi-major axes as there is in the other models, which makes the extent of the support for the inner bar less well defined in model 05E. The last loop supporting the inner bar (the 8th loop) has also the longest semi-major axis, and therefore serves as the estimate of the bar length.

As in models 01 and 05, the loops supporting the inner bar in model 05E (loops 2-8, the first loop is excluded because of pulsation) maintain coherence throughout rotation (see Figure 3a). When the angle between the bars in the imposed potential is $45^{\circ}$, the mean $\mathrm{PA}$ of loops supporting the inner bar is $50.2^{\circ}$, with an average error of $1.5^{\circ}$. This implies that the acceleration of the inner bar in this model is consistent with that in models 01 and 05 . The loops in model $05 \mathrm{E}$ are the roundest of all models, as shown in Figure 3g. The eccentricity of the loops (Figure 4) implies that the inner bar in model 05E also seems to undergo less pulsation than in any of the other models.

\subsubsection{Model 04}

In models 04 to $02 \mathrm{E}$, the pattern speed for the inner bar successively decreases from its value in model 01 . In model 04 it is $100 \mathrm{~km} \mathrm{~s}^{-1} \mathrm{kpc}^{-1}$. The extent of the support for the inner bar in this model is greater than in model 01. The last loop supporting the inner bar in model 04 is the 10th loop, but among these loops the 9 th loop serves as the estimate of the bar length, because it has the longest semi-major axis.

As can be seen in Figure 3d and Figure 4, the loops supporting the inner bar in model 04 (loops 3-10, since as in the models above, two inner-most orbits, dominated by pulsation, are excluded from loops supporting the inner bar) still behave coherently throughout rotation. The average PA of loops supporting the inner bar, when the angle between the bars in the imposed potential is $45^{\circ}$, is $51.4^{\circ}$ with an average error of $2.5^{\circ}$. This implies the acceleration of loops in model 04 slightly larger than in the models above, but by no more than a typical measurement error. Figure 3j shows that the loops supporting the inner bar in model 04 are more eccentric than in model 01. The loops supporting the inner bar in model 04 also undergo a lot more pulsation than in model 01 (Figure 4).

\subsubsection{Model 03}

The pattern speed of the inner bar for model 03 is 90 $\mathrm{km} \mathrm{s}^{-1} \mathrm{kpc}^{-1}$. There is a gap between loops 8 and 9, which corresponds in the ring-width diagram (Figure 1c) to the region of the inner arch, where its dark spine is apparently missing. We could not find good approximations to the loops in this region. This means that even if a more detailed search succeeds in finding the loops there, regular orbits are not trapped well there, hence the support for the inner bar is strongly reduced. Nevertheless, the extent of the inner bar, in terms of support from the $x_{2}$ orbits, is still greater than in the models above. The last loop supporting the inner bar is the 8th loop, and because of the gap past it, where we could not find loops, it has the longest semi-major axis among 


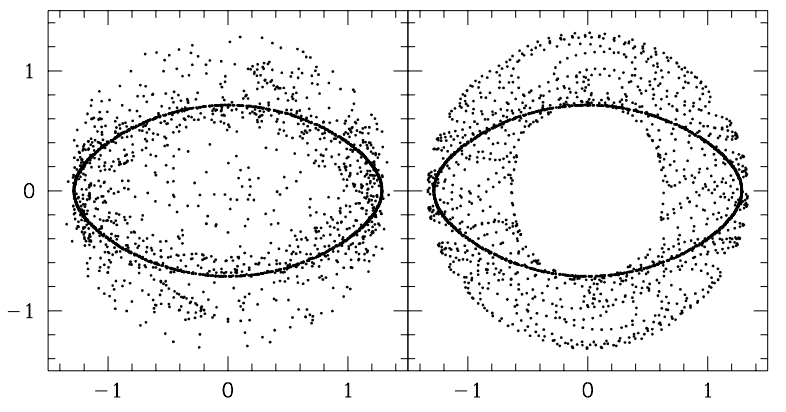

Figure 5. Representations of three orbits in the potential of two independently rotating bars from model 02 , plotted at the moments when the two bars are aligned, for 1200 consecutive alignments of the bars. The solid line in both panels marks the 8th loop in this model. The dots in the left-hand panel mark the representation of an orbit with the same initial velocity and the initial position equal to 0.97 of that of the orbit that is represented by the 8 th loop. The dots in the right-hand panel mark the representation of an analogous orbit whose initial position is now multiplied by 1.03 . Units on axes are in kpc.

these loops. However, the extent of the bar may still be slightly larger if a loop supporting this bar were found past the 8th loop.

Contrary to the models analysed so far, the loops supporting the inner bar in model 03 do not behave coherently throughout rotation: when the angle between the bars in the imposed potential is $45^{\circ}$, in Figure 2 there is a twist in loops supporting the inner bar, reflected by a change in the PA in Figure 3e. One can see that the deeper inside the bar the loop is located, the more it accelerates. An upturn in the PA for the inner-most loop appears now to be more consistent with the trend exhibited by the other loops, but for the sake of consistency with the other models we still exclude the inner-most loop from the loops supporting the inner bar. In model 03, the mean PA of the loops supporting the inner bar (loops 2-8) is larger than in the models above (Table 1), hence the inner bar in this model undergoes more acceleration. The loops supporting the inner bar in model 03 are more eccentric compared to the models above and they imply greater pulsation of the inner bar than in the models above, but this pulsation is no longer consistent among the loops (Figure 4).

\subsubsection{Model 02}

The pattern speed of the inner bar in model 02 is 80 $\mathrm{km} \mathrm{s}^{-1} \mathrm{kpc}^{-1}$. The last among the loops supporting the inner bar is the 9 th loop, but among them the 8th one has the longest semi-major axis and therefore serves as the bar length estimate. In the ring-width diagram in Figure $1 \mathrm{~b}$, the initial conditions for loops 8-10 in model 02 are taken from a ridge-like feature in the right leg of the inner arch. This ridge departs upwards from the course of the dark spine of the inner arch on the lower right. On the right of this ridge, there are light grey areas indicating poorly trapped trajectories, and on the left there is white area indicating chaotic trajectories with initial conditions immediately next to those of the loops. Finding loops on this ridge is unexpected since stable double frequency orbits should be well surrounded by trapped trajectories. In fact in model 03, for corresponding initial conditions (between loops 8 and 9 there) we were not able to find loops at all: trapping of trajectories was increasing when moving to the left in the ring-width diagram in this region, but then the trajectories were abruptly becoming chaotic before they converged on loops. We checked the appearance of orbits around the ridge in model 02: Figure 5 shows the 8th loop, and points sampled from the trajectories whose initial positions are just 3\% smaller and larger than that of the loop (i.e. come from adjacent pixels in the ring-width diagram). As expected, the trajectory with initial conditions from the left of the ridge (Figure 5, left-hand panel) looks chaotic, while the trajectory whose initial conditions are to the right of the ridge (Figure 5, right-hand panel), although regular, does not align with the loop, and hence it does not support the bar. Therefore the outermost loops of the inner bar (loops 7-9) in model 02 provide essentially no support for this bar in terms of trapped trajectories. The resolution of the ring-width diagram is barely sufficient to locate these loops, hence in Figure 2 they appear as a collection of noticeably scattered points.

The twisting of the loops in the inner bar is even more dramatic in model 02 than in model 03. In Figure 2, these loops form a spiral rather than a bar, when the angle between the bars in the imposed potential is $45^{\circ}$. As in model 03, the inner-most orbit is excluded from loops supporting the inner bar. Loops supporting the inner bar in model 02 strongly pulsate but the change in eccentricity is even more inconsistent among them than in the models analysed above, as shown in Figure 4.

\subsubsection{Model 02E}

The pattern speed for the inner bar in model $02 \mathrm{E}$ is $70 \mathrm{~km} \mathrm{~s}^{-1} \mathrm{kpc}^{-1}$. This is a new model, not presented in MA08, and it extends the range of models from MA08 to lower pattern speeds. The ring-width diagram for this model, presented in Figure 1a, looks very different from those for all the other models analysed in this paper. The loops, presented in the bottom row of Figure 2, are also significantly different. The innermost two loops have the initial conditions taken from the $V$-shaped feature branching from the outer arch in Figure 1a. These loops seem to rotate in the opposite direction to the two bars. There is a region of small ring-widths between the left leg of the outer arch and the inner arch. The two orbits recovered there map onto loops 3 and 4 which seem to be dominated by pulsation rather than rotation. The inner arch in Figure 1a looks also different than in the models above: its left leg is considerably shortened and occupies the area that is chaotic in the ring-width diagrams of all the other models. The inner loops recovered in this area seem to be oriented perpendicularly to the inner bar, as if they originated from the $x_{2}$ orbits in the inner bar. None of the loops in model 02E support the inner bar and therefore they are not analyzed further in Figures 3 and 4 .

\subsection{Dependence of inner bar's properties on its pattern speed}

\subsubsection{The length of the inner bar}

In the fourth column of Table 1, we list the length of the semi-major axis of the inner bar, determined with the method described in Section 2.3 as the maximum extent of the orbits that support it. Despite our estimation of 


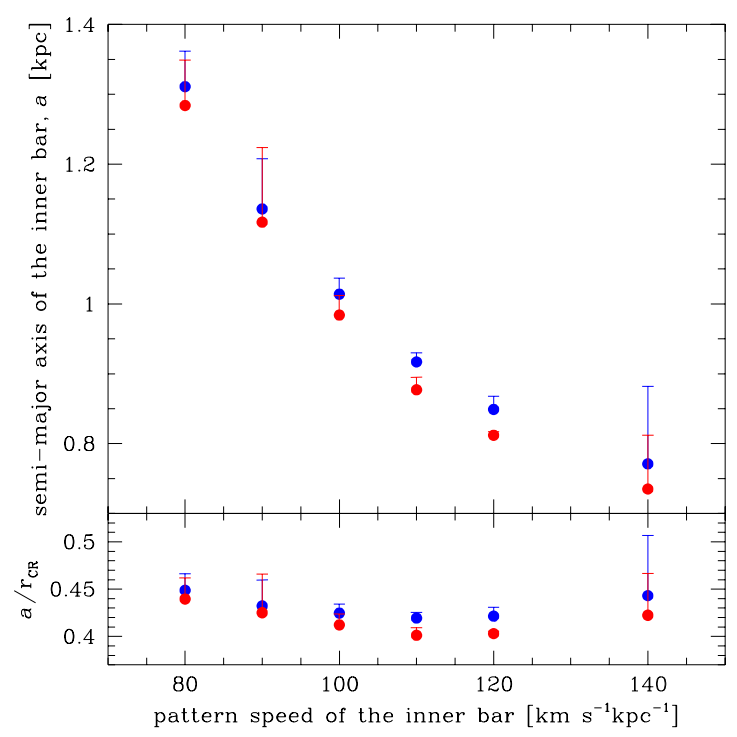

Figure 6. For each of the models $02-05 \mathrm{E}$, characterized by the pattern speed of the inner bar in each model, plotted on the horizontal axis, we plot the length of the inner bar as determined from the extent of the loops that support it (upper panel), and the ratio of this length to the corotation radius of the inner bar in each model (lower panel). Red points represent measurements at the moment when the two bars are parallel, and blue points - when the bars are perpendicular. Error bars mark upper limits for the length of the bar estimated following the method in Section 2.3. The points, from left to right, correspond to models $02,03,04,01$, 05 and $05 \mathrm{E}$.

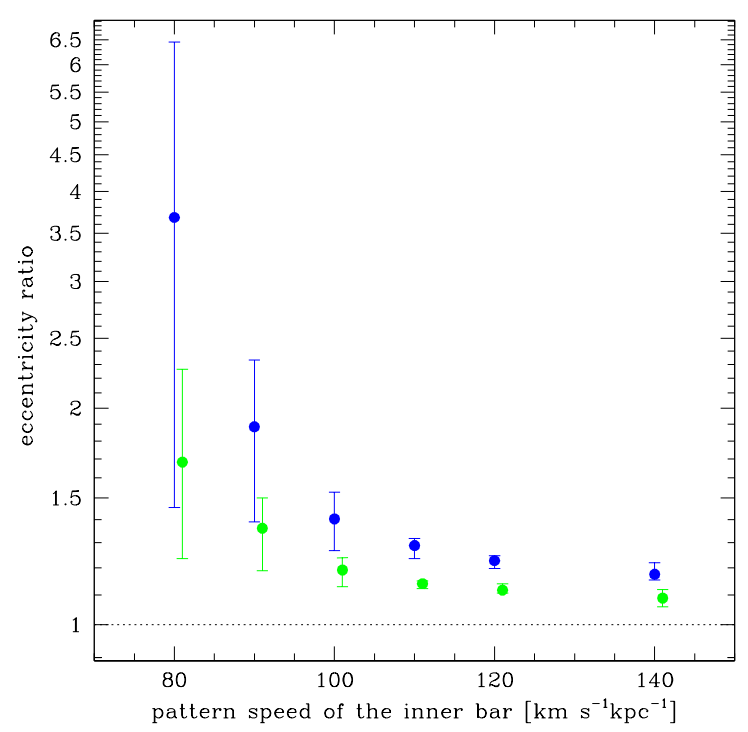

Figure 7. For the loops supporting the inner bar in each of the models $02-05 \mathrm{E}$ (characterized by the pattern speed of the inner bar in each model, that is plotted on the horizontal axis) we plot the average ratio of the eccentricity at the moment when the two bars are perpendicular to the eccentricity when the two bars are parallel (blue points) and the average ratio of the eccentricity at the moment when the the angle between the two bars in the assumed potential is $45^{\circ}$ to the eccentricity when the two bars are parallel (green points). Error bars mark the range of this ratio for all the loops that support the inner bar. The points, from left to right, correspond to models $02,03,04,01,05$ and 05E. For clarity, data plotted in green are slightly shifted to the right from data plotted in blue. the bar length being approximate, there is a clear trend in the models. The extent over which the inner bar is supported by orbits that align with it evidently decreases with the pattern speed of this bar, as we already noticed when studying individual models in Section 3.1. In the upper panel of Figure 6 we plot the implied length of the inner bar as a function of its pattern speed, measured at the moments when the two bars are parallel and perpendicular to each other, as this length varies slightly when the bars rotate through each other. It is clear that the extent of orbital support for inner bars of lower pattern speed extends further out.

When the implied length of the semi-major axis of the inner bar is plotted in units of its corotation radius (Figure 6, lower panel, and Table 1, column 5), it exhibits no clear trend with the pattern speed, and in all models the inner bar has orbital support out to $40 \%-44 \%$ of its corotation radius. The ratio of the implied bar size to its corotation radius is therefore remarkably constant. This result confirms the prediction of MS00, already verified by numerical models (e.g. Heller et al. 2007, Shen \& Debattista 2009): the inner bar in a doubly barred system is unlikely to extend to its corotation.

\subsubsection{The eccentricity and the pulsation of the inner bar}

In the sixth column of Table 1, we list the largest axial ratio of the loops supporting the inner bar at the moment when the two bars are parallel. This axial ratio is the indicator of the eccentricity of the bar. As we already noticed when studying individual models in Section 3.1, the eccentricity of the inner bar, implied by shapes of the loops that support it, decreases with the bar's pattern speed: the faster rotating bar becomes rounder.

In all models presented in this paper orbital support of the inner bar indicates that this bar should pulsate as it rotates through the outer bar. The inner bar becomes rounder as it aligns with the outer bar, while its eccentricity is largest when the two bars are perpendicular (see Figure 3, right-hand panels), as already predicted by MS00. The pulsation of the inner bar, quantified by the average ratio of eccentricities of the loops at the moments when the two bars are parallel and perpendicular, decreases with the pattern speed of the bar (Table 1, column 7). Thus the lower the pattern speed of the inner bar, the more eccentric that bar is and the more it pulsates. In Figure 7 we plot the average ratio of eccentricities of the loops between the moments when the two bars are parallel and perpendicular, as well as between the moments when the two bars are parallel and at $45^{\circ}$. It can be seen that the average eccentricity of the loops when the bars are perpendicular increases from its value when they are parallel by about twice as much as it increases when the angle between the two bars is $45^{\circ}$. It is worth pointing out that the inner bar pulsates even when it is very round: the largest axial ratio of the loops supporting the inner bar in model $05 \mathrm{E}$ is only 1.5 when the bars are parallel, but it still increases by $18 \%$ to 1.78 when the bars are perpendicular.

For the models with the highest inner bar pattern speeds (models 01, 05 and 05E), the loops pulsate only slightly and in a coherent way (see Figure 4). For models with lower pattern speeds of the inner bar, and model 02 in particular, the average pulsation is more extreme and there is more variation in the pulsation of individual 


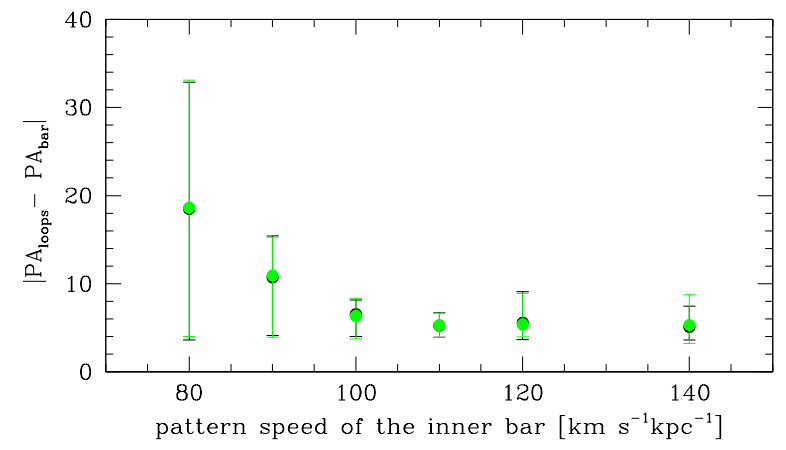

Figure 8. For each of the models 02-05E (characterized by the pattern speed of the inner bar in each model, which is plotted on the horizontal axis) the dots mark the average offset in the PA of the major axis of the loops that support the inner bar from the PA of the uniformly rotating major axis of the inner bar in the imposed gravitational potential at the moments when the angle between the two bars in the imposed potential is $45^{\circ}$ (green) and $135^{\circ}$ (black). The maximum and minimum of this offset is marked by the error bars. The points, from left to right, correspond to models 02,03 , 04, 01, 05 and 05E.

loops (Figure 7). Thus a slowly rotating inner bar does not pulsate coherently: when the eccentricity of its outermost loops increases by a mere $50 \%$ the eccentricity of its inner loops can increase by factors larger than six.

\subsubsection{The non-uniform rotation of the inner bar}

As predicted from the orbital structure of double bars (MS00) and confirmed by numerical models (Debattista \& Shen 2007), the inner bar in a doubly barred galaxy does not rotate uniformly, but its angular velocity is highest when the bars are aligned and lowest when the bars are perpendicular. The same is implied by the orbital models presented here: the loops supporting the inner bar align with the uniformly rotating major axis of the bar in the assumed gravitational potential when this bar is parallel or perpendicular to the outer bar, but they lead this axis when the inner bar departs from the alignment with the outer one, and trail it on the way back to the alignment (see Figure 3, left-hand panels). In particular, when in the assumed gravitational potential the angle between the major axes of the two bars is $45^{\circ}$, the $\mathrm{PA}$ of the major axis of the loops supporting the inner bar is offset from this angle to a larger value. This offset is listed in the last column of Table 1. It indicates the amplitude of the variation of the pattern speed of the inner bar. If for simplicity one assumes that this variation is sinusoidal, then the actual pattern speed of the inner bar, $\Omega_{S}^{v}(t)$, relates to the assumed constant pattern speed, $\Omega_{S}$, by $\Omega_{S}^{v}(t)=\Omega_{S}\left(1+2 \epsilon \cos \left[2 \Omega_{S} t\right]\right)$, where $\epsilon$ in radians is the PA offset listed in Table 1.

In Figure 8, we plot the PA offset when the angle between the inner and the outer bar is $45^{\circ}$ and $135^{\circ}$. In addition to the average PA offset, we plot its maximum and minimum among the loops supporting the inner bar in each model, represented by error bars. The offset for $45^{\circ}$ and $135^{\circ}$ looks the same, because time is reversible in the equation of motion. Small range between the minimum and maximum offset indicates that the loops remain aligned, and therefore the non-uniform rotation of the bar remains coherent. Thus fast inner bars (models $04,01,05$ and $05 \mathrm{E}$ ) rotate coherently. When the angle

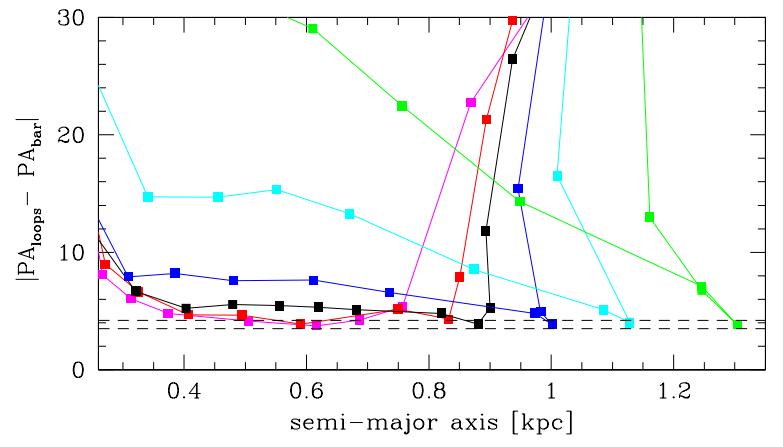

Figure 9. The PA offset, averaged between the moments when the angle between the bars in the assumed gravitational potential is $45^{\circ}$ and $135^{\circ}$, plotted for the loops in model 05E (pink), 05 (red), 01 (black), 04 (blue), 03 (cyan) and 02 (green), against their semimajor axes measured at those moments. Each loop is marked by a filled square, with lines that connect them reflecting the sequence along the arch in Figure 1. In each model, the minimal PA offset falls between the two black dashed lines drawn for $3.5^{\circ}$ and $4.2^{\circ}$.

between the bars in the assumed potential is $45^{\circ}$, they lead the inner bar in the assumed potential by $5^{\circ}-7^{\circ}$, which implies that the pattern speed of the inner bar varies by $18 \%-24 \%$ around its nominal value.

Also in Figure 8 it can be seen that slowly rotating inner bars (models 02 and 03) have the PA offset on average larger, and the range between its minimum and maximum increases as the pattern speed of the inner bar is lowered. This means that the slower the inner bar rotates, the less uniform and less coherent its rotation is. On average, the pattern speed of the inner bar is predicted to vary around its nominal value by $38 \%$ in model 03 and by $65 \%$ in model 02 . However, as can be seen in the left-hand panels of Figure 3, in both models the PA offset increases towards the centre, hence the rotation is least uniform in the central parts of the inner bar. Inner loops in model 02 have the PA offset above $25^{\circ}$, which means that their pattern speed varies by a factor of a few (the no longer adequate sinusoidal approximation gives the amplitude of $90 \%$ ).

On the other hand, rotation in the outer parts of the slowly rotating inner bars is as uniform as in the fast bars. In Figure 9, we show the PA offset (averaged between the moments when the angle between the bars in the assumed gravitational potential is $45^{\circ}$ and $135^{\circ}$ ) for individual loops in all the models. Its value at the ends of the inner bar in models 02 and 03 (1.3 and $1.12 \mathrm{kpc}$, respectively) is as low as the lowest value for models 05 and $05 \mathrm{E}$ - just about $4^{\circ}$. It corresponds to the variation of inner bar's pattern speed by just 14\%. Interestingly, in Figure 9 can also be seen that the minimal PA offset in each model is bound within a very small range: $3.5^{\circ}-4.2^{\circ}$, indicated by the two dashed lines in that figure.

Given the analysis above, the rotation of the inner bar should be most coherent in models 05 and $05 \mathrm{E}$. However, in Figure 8 the range of the PA offset in these models is larger than in model 01, whose inner bar rotates more slowly. This is because the innermost loops that we included into the inner bar in models 05 and $05 \mathrm{E}$ (red and pink squares furthest to the left in Figure 9) have no counterpart in model 01 , while being most likely perturbed by the inner ILR of the outer bar. If we exclude these loops, the range of the PA offset for all the loops 
supporting the inner bar in models 01,05 and $05 \mathrm{E}$ is just $3^{\circ}$ in each case, hence the inner bar in these three models rotates very coherently.

\section{DISCUSSION}

\subsection{The validity of orbital structure studies}

The major drawback of orbital studies is that they impose the potential without checking whether the stars put on orbits in this potential can reproduce the imposed potential. However, orbital studies form only a first step in dynamical analysis, by providing a framework to fully self-consistent models, like in N-body simulations. Their role is to identify orbits that are the backbone of the various galactic structures, conditions for the existence of these orbits, and to estimate the extent of these orbits as a function of parameters of the potential. These studies allow a quick exploration of the parameters of the system analyzed, as these parameters are part of the imposed potential.

The fruitfulness of orbital studies can be seen on the example of our understanding of the structure of classical single bars. As stated by Sellwood \& Wilkinson (1993), by the time of that review, only one self-consistent solution for a rapidly rotating two-dimensional bar existed to their knowledge (see section 4.9.1 there). Thus our insight into bar dynamics came mostly from orbital studies, which lack self-consistency: the finding of Contopoulos (1980) that bars should end within their corotation was confirmed by detailed studies of Contopoulos \& $\mathrm{Pa}$ payannopoulos (1980), which assume the bar potential that actually extends beyond the corotation. Athanassoula et al.(1983) related inner ring structures observed in some galaxies to stable circular periodic orbits near the corotation, using bars extending to twice their corotation radius in order to enhance the orbital response of interest. The orbital theory provided explanation for the existence and location of the lobes or 'ears' at the ends of the bar (Teuben \& Sanders 1985), and the loops of the near-IR isophotes (Patsis et al. 1997) without modeling these structures in a self-consistent way. Pfenniger (1984) explained the rectangular aspect of the bulge observed in edge-on galaxies by the 4/1 vertical resonance, and Bureau \& Athanassoula (1999) proposed diagnostics to determine the viewing angle with respect to the bar in an edge-on disk based on signatures of orbit families in the position-velocity diagrams. They were all using models that lack self-consistency. The method of our analysis is parallel to that of Patsis et al. (2003), who studied the orbital response to varying pattern speed of an imposed bar of a fixed length and axial ratio, and found that outer boxiness of the bar is favoured by fast pattern speeds, while a slowly rotating bar is surrounded by almost circular orbits.

The success of orbital analysis can be partially explained by the fact that the orbits can be seen as a sum of oscillations in the gravitational potential, and therefore the orbital response depends more on resonances among the frequencies involved than on the particular shape of the gravitational potential. Several results of orbital studies of double bars (MS00) have already been confirmed by fully self-consistent N-body simulations (Debattista \& Shen 2007; Shen \& Debattista 2009). Due to the presence of an outer bar, the inner bar pulsates (being thinnest when the bars are perpendicular and thickest when parallel), accelerates (spending more time nearly perpendicular to the outer bar than nearly parallel to it), and ends well within its own corotation. These results come from orbital studies of double barred galaxies, in which a potential of two rigidly rotating bars is imposed. Future work, in which imposed potentials do not require the two bars to rotate rigidly, should bring such orbital studies closer to self-consistency.

\subsection{Is orbital support better in fast or slow inner bars?}

Orbital models analyzed in this paper show that the lower the pattern speed of the inner bar, the larger the radial extent of the orbits that support it. However, at lower pattern speeds these orbits trap around themselves regular trajectories less efficiently than at higher pattern speeds. It is especially true for orbits supporting outer parts of the inner bars, and the extreme example is shown in Figure 5. This means that although at low pattern speeds the bar can be longer, it can drag with itself only a small fraction of stars, as it does not trap stellar trajectories well. On the other hand, increasing the pattern speed of the inner bar leads to more efficient trapping of trajectories, but at high pattern speeds, orbits support only short and round inner bars. Thus there is a tradeoff between the length of the inner bar and the support of this bar by regular trajectories. Both extremes, at the lowest and highest pattern speed of the inner bar are possible, as well as the intermediate solutions between them. However, there is no preferred pattern speed for which the orbital support is 'best' in any sense. In particular, we confirm the finding of MA08 that the inner bar in resonant coupling with the outer bar is not preferred in any way.

In the high pattern speed limit (model 05E), the orbital structure implies inner bar that is short, round and can trap a good fraction of stars. As pointed out in Section 3.1 .3 , its end is not well defined, and the transition region to the outer bar may look like an isophotal twist (Figure 2 , top panels). The size of this transition zone increases with inner bar's pattern speed (see left-hand columns of Figure 3 for models 05 and $05 \mathrm{E}$ ), and one may expect that at still higher pattern speeds the morphology in the inner bar region is dominated by the isophotal twist.

In the low pattern speed limit (model 02), the orbital structure implies inner bar that is long, eccentric and can trap only a small fraction of stars. Such bar has a well defined end in terms of orbits that support it, but as these orbits do not trap stars well, the bar's end may not be defined that well in stellar distribution. Further decreasing of pattern speed of the inner bar erases the supporting orbits abruptly and completely. Thus there is a well defined lowest possible, critical pattern speed of the inner bar. For pattern speeds lower than that, there are still regular orbits within the inner bar, but their appearance is dramatically different, and they no longer support the shape of the inner bar. Most of them remain perpendicular to the inner bar. If the presence of such orbits were a mere consequence of this bar having an ILR, they should be also present in models 04, 03 and 02, as all these models have an ILR (see fig.9 in MA08). We searched for such orbits in those models, but we did not find any. Also, if the structure of the inner and the outer bar were similar, families of orbits both parallel and 
perpendicular to the bar should coexist within the same model, which is not the case in our models. The abrupt disappearance, at low pattern speeds, of orbits aligned with the inner bar, and their replacement throughout the extent of the bar by orbits perpendicular to it, which were absent at higher pattern speeds, reflects the nonlinear interaction between the bars in doubly barred galaxies. The value of the critical pattern speed found in this paper is specific to the set of models considered here, and it is not clear by what physical mechanism it is determined. It is close to twice the pattern speed of the outer bar, and one can hypothesize that the low-order resonance between the bars destroys the support of the inner bar. However, no transition in orbital structure is seen when the pattern speed of the inner bar is three times that of the outer bar.

\subsection{Possible dynamical interpretation of the observed morphologies}

Recently a method has been proposed for extracting inner bars from observations of early-type spirals by modelling and subtracting the disk, bulge and the outer bar. The results for two galaxies have already been presented (Erwin 2010). The two extracted inner bars are quite different from each other. One (in NGC 1543) contains a small fraction of the total stellar light (4\%), its eccentricity is high (axial ratio of 4 ), and its extent rather well defined, with isophotes aligned throughout the bar. The other bar (in NGC 2859), to the contrary, contains a much larger fraction of the total light (10\%), its eccentricity is lower (axial ratio of 2), and its outer part smoothly turns into a region of twisted isophotes outside it, which at larger radii become round and similar to those of the subtracted components of the galaxy in the same region.

Based on our knowledge of single bars, one should not necessarily expect correlations between the three characteristics above (eccentricity, mass, and outer isophotal shape). It is therefore significant that in the observations they correlate in the same way as in our two models of extreme pattern speeds. Orbits supporting the slowly rotating inner bar in our models map onto loops that are eccentric, hence large axial ratio of such a bar, but they do not trap stars well, hence small mass fraction in the bar. We also showed that because of its orbital structure the extent of a slow bar is well defined. The inner bar in NGC 1543 is eccentric, contains small mass fraction and has a well defined extent, which suggests that it rotates slowly in the sense that its pattern speed is close to the lowest possible one.

On the other hand, in our models orbits supporting the inner bar of high pattern speed map onto loops that become increasingly round as the pattern speed of the bar increases, therefore the observed axial ratio of that bar should be small. These orbits trap stars very efficiently, hence the majority of the stars within the extent of the bar follow that bar and the mass fraction in the bar is large. Outer loops of fast rotating inner bars become monotonically rounder as their major axes increase, and enter a transition region that looks like an isophotal twist, so that the end of the bar is not well defined. The inner bar of NGC 2859 is less eccentric than in NGC 1543 , contains a high mass fraction and is surrounded by an isophotal twist which suggests that the angular veloc-
Table 2

Morphological characteristics of slow and fast inner bars in doubly barred galaxies

\begin{tabular}{lll}
\hline & slow inner bar & fast inner bar \\
\hline mass fraction & small & large \\
eccentricity & large & small \\
end of bar & well defined by & unclear, turns into \\
& aligned isophotes & twisted isophotes \\
\hline
\end{tabular}

ity of the inner bar in NGC 2859 is considerably larger than its lowest dynamically possible value, and that the structure of this inner bar is close to the structure in models of fast inner bars presented here.

Thus, we propose that from the morphology of extracted inner bars we can discriminate between slow bars, whose pattern speed is close to the minimal dynamically possible value, and fast bars, whose pattern speed is considerably above this limit (possibly two times higher). This distinction can be based on the characteristics listed in Table 2. Note that by slow and fast inner bars we do not mean the ratio of the length of the bar to its corotation radius, which in our models is virtually constant. Our slow bars have pattern speeds in a range whose lower limit is set by no orbital support for the inner bar, while our fast bars become increasingly rounder as their angular velocity increases, and eventually they cannot be distinguished from axisymmetric components of the galaxy.

\section{CONCLUSIONS}

In this paper, we studied the orbital support of the inner bar in seven models of double-barred galaxies, with the angular velocity of the inner bar different in each model. The aim of this work was to analyze how this support changes with the bar's pattern speed.

The new result of this analysis is that the pattern speed of the inner bar cannot be arbitrarily low. When this pattern speed drops below a certain threshold, the family of loops that support the inner bar is completely wiped out. It is instantly replaced by a family of loops perpendicular to that bar, possibly related to the $x_{2}$ orbits in the inner bar. We find no models in which both families of loops coexist. In addition to the lower limit for the angular velocity of the inner bar, set by an abrupt destruction of orbits that support it, there is a soft upper limit, which comes from the bar becoming increasingly rounder as its angular velocity increases, so that it no longer is a distinct dynamical feature. These limits apply only to double bars that rotate in the same direction, as only such bars were considered in this paper. Maciejewski (2008) showed that in counter-rotating double bars, inner bars are supported by loops corresponding to a different orbital family $\left(x_{4}\right)$, and their dynamically possible angular velocities may not be limited in a similar way.

The models presented in this paper show that the pattern speed of the inner bar has a significant impact on its structure and dynamics. Earlier studies were limited to single models of double bars, and this work extends what we already know based on those studies. In particular, the predictions of orbital models by MS00, verified by the $N$-body simulations of Debattista \& Shen (2007) and Shen \& Debattista (2009), are extended as follows:

1. Confirming that the inner bar should end well 
within its corotation radius, we show that the orbital support of the inner bar extends further out in radius for lower pattern speeds than it does for higher ones. However, lower pattern speed means larger corotation radius, and the ratio of the extent of orbital support of the inner bar to its corotation radius remains remarkably constant for the models constructed here. For low pattern speeds of the inner bar, double-frequency orbits supporting outer parts of that bar trap trajectories that do not occupy large volumes of phase-space, hence provide only a limited support for the bar.

2. Confirming that the inner bar pulsates as it rotates through the outer bar, we show that the lower the pattern speed of the inner bar, the more eccentric the loops that support it, and the more they pulsate as the bars rotate through each other.

3. Confirming that the rotation of the inner bar in a doubly barred galaxy is not uniform, we show that when the angular velocity of the inner bar is small, no more than $30 \%$ above the threshold below which the loops that support it are wiped out, these loops do not rotate coherently: the angular velocity of the inner loops varies severely, much more than that of the outer loops. On the other hand, faster inner bars rotate coherently: the loops that support the inner bar remain aligned within a few degrees of each other as this bar rotates through the outer bar. The rotation of such bars is quite uniform as well: the common angular velocity of the loops varies only by about $20 \%$ around its average value.

We find no dynamically preferred pattern speed of the inner bar. In particular, there is no evidence of minimizing chaos at resonant coupling between the two bars. However, there is a trade-off in the properties of the bar instead. At large pattern speeds, loops supporting the inner bar trap trajectories that occupy large volume of phase-space, so the inner bar can contain a large fraction of stars, but it is also short and round. At small pattern speeds, the inner bar is longer, more eccentric, but it pulsates and accelerates more, and the volume of chaotic zones increases, hence the bar cannot trap enough stars to make its mass high. This correlation between mass, eccentricity and the isophotal shape seems to be reflected by the recent observational decomposition of inner bars in doubly barred galaxies.

We would like to thank Peter Erwin for commenting on this work, and Lia Athanassoula for inspiring discussions. This work was partially supported by the Polish Committee for Scientific Research as a research project 1 P03D 00726 in the years 2004-2007. WM acknowledges Academic Fellowship EP/E500587/1 from Research Councils UK.

\section{REFERENCES}

Athanassoula, E., Bienayme, O., Martinet, L., \& Pfenniger, D., 1983, A\&A, 127, 349

Bureau, M., \& Athanassoula, E., 1999, ApJ, 522, 686

Buta, R., \& Crocker, D.A., 1993, AJ, 105, 1344

Debattista, V. P. \& Shen, J. 2007, ApJ, 654, L127

de Vaucouleurs, G., 1975, ApJS, 29, 193

Contopoulos, G., 1980, A\&A, 81, 198

Contopoulos, G., \& Papayannopoulos, Th., 1980, A\&A, 92, 33

Corsini, E. M., Debattista, V. P., \& Aguerri, J. A. L., 2003, ApJ, 599, L29

Erwin. P., 2010, Mem. della Soc. Astr. Italiana, in press, (arXiv:0908.0909)

Erwin, P., \& Sparke, L., 2002, AJ, 124, 65

Friedli, D., \& Martinet, L., 1993, A\&A, 277, 27

Heller, C., Shlosman, I., \& Athanassoula, E., 2007, ApJL, 657, L65

Laine, S., Shlosman, I., Knapen, J. H., \& Peletier, R. F., 2002, ApJ, 567, 97

Maciejewski, W., 2008, Astron. Soc. of the Pacific Conf. Ser., 396, 367

Maciejewski, W., 2010, Mem. della Soc. Astr. Italiana, in press, (arXiv:0903.2485)

Maciejewski, W., \& Athanassoula, E., 2007, MNRAS, 380, 999 (MA07)

Maciejewski, W., \& Athanassoula, E., 2008, MNRAS, 389, 545 (MA08)

Maciejewski, W., \& Sparke, L., 1997, ApJ, 484, L117

Maciejewski, W., \& Sparke, L., 2000, MNRAS, 313, 745 (MS00)

Moiseev, A. V., 2010, Mem. della Soc. Astr. Italiana, in press, (arXiv:0811.3527)

Moiseev, A. V., Valdes, J. R., \& Chavushyan, V. H., 2004, A\&A, 421,433

Patsis, P. A., Athanassoula, E., \& Quillen, 1997, ApJ, 483, 731

Patsis, P. A., Skokos, Ch., \& Athanassoula, E., 2003, MNRAS, 342,69

Pfenniger, D., 1984, A\&A, 134, 373

Sellwood, J. A., \& Wilkinson, A., 1993, Rep. Prog. Phys., 56, 173

Shen, J. \& Debattista, V. P, 2009, ApJ, 690, 758

Teuben, P. J., \& Sanders, R. H., 1985, MNRAS, 212, 257 